Revista de Psicología de la PUCP. Vol. XXII, 1, 2004

\title{
Motivación en línea y resultados del aprendizaje en estudiantes universitarios
}

\author{
María de los Ángeles Maytorena Noriega ${ }^{1}$, Daniel González Lomelí ${ }^{2}$ \\ y Sandra Castañeda Figueiras ${ }^{3}$ \\ Universidad de Sonoral Universidad Nacional Autónoma de México
}

\begin{abstract}
El objetivo del trabajo fue someter a prueba una estructura multifactorial de la evaluación en línea de la motivación sobre la realización de una tarea académica específica en estudiantes de licenciatura. Los resultados indican que la motivación con la que inician la tarea explica el $12 \%$ de la varianza de la puntuación total obtenida en la prueba de metodología y ésta a su vez, explica el $17 \%$ de la varianza del nivel de motivación posterior a la ejecución. El modelo de evaluación en línea desarrollado permitirá la prescripción instruccional a la medida de las necesidades del alumno con el propósito de facilitar el aprendizaje y favorecer el éxito académico.

Palabras clave: motivación, evaluación en línea, modelo estructural.
\end{abstract}

\section{Line motivation and learning results in university students}

The aim of this study was to test a multifactor structure for in-line-evaluation of motivation on specific tasks in university students. Results showed that the initial motivation explained $12 \%$ of the variance of total punctuation of the methodology test and test execution explained $17 \%$ of the ending motivation. Therefore, in-line-evaluation model allows instructional prescription to students needs in order to facilitate their learning and academic success.

Key words: motivation, in-line evaluation, structural model.

1 Profesora del Departamento de Psicología en la U. de Sonora. Maestra en Innovación Educativa por la U. de Sonora. Correo electrónico: mmaytorena@psicom.uson.mx. Profesor titular del Departamento de Psicología de la U. de Sonora. Doctor en Psicología por la UNAM. la Sociedad Iberoamericana de Pensamiento y Lenguaje. Doctora en Psicología Experimental. 

Avances contemporáneos en el entendimiento de la cognición humana han abierto la caja negra de la actividad cognitiva constructiva (Glaser \& Baxter, 2002) y la de sus asociados autorregulatorios (Boekaerts, Pintrich \& Zeidner, 2000). Aportan evidencia significativa sobre mecanismos determinantes del éxito académico que hacen posible extender la comprensión, el razonamiento, la solución a problemas y la autorregulación del aprendizaje. Conforman un marco teórico sólido, empíricamente sustentado, capaz de servir de base para diseñar ambientes de aprendizaje más poderosos y útiles. Sin embargo, y aun cuando en teoría, el interés por conocer y operar mecanismos que controlan el aprendizaje constituye un loable fin práctico - el que todos reciban una buena educación - la realidad de alumnos y profesores se encuentra muy distante de lo deseado, las actividades educativas ordinaris no muestran la mejora deseada en la mayoría de los niveles educativos (Castañeda, Lugo, Pineda \& Romero, 1998; De Corte, 1995).

En este trabajo se aborda investigación que traduce premisas teóricas acerca de la cognición humana en un marco de trabajo útil para derivar herramienta tecnológica. En concordancia con Castañeda (en prensa), la asunción subyacente a la investigación plantea que la aplicación de tales premisas juega un papel central en el diseño de herramientas para la comunidad educativa y que los esfuerzos por asegurar una fuerte relación entre la investigación y la práctica puede apoyar cambios deseados en la práctica educativa ordinaria. La autora reconoce que la mejora educativa requiere de múltiples acciones, entre otras principales, evaluar y fomentar componentes que aseguren aprendizajes establecidos por la comunidad educativa, internacional y nacional, como altamente deseables. 
María de los Ángeles Maytorena N., Daniel González L. y Sandra Castañeda F.

Así, los desarrollos actuales en evaluación y fomento del aprendizaje eficiente concretizan su interés en la actividad cognitiva constructiva autorregulada, tópico reconocido como central en la formación académica contemporánea.

La actual evaluación cognitiva de resultados de aprendizaje se realiza mediante marcos multicomponenciales de medición (Castañeda, 1993, 1998, 2002) que utiliza análisis funcional de competencias y cognitivo de tareas para descomponer, recursivamente, los elementos que componen una tarea compleja. Los productos de ambos análisis apoyan la identificación de posibles fallas en el estudiante (dada una previa y secuenciada organización, por bloques, de conocimientos, habilidades y actitudes), tanto como la prescripción de la instrucción requerida, dentro de un contexto integrado e interactivo entre evaluación e instrucción (Castañeda, 1993). Dado que la medición es un proceso inferencial, el propósito de utilizar marcos multicomponenciales es asegurar que las regularidades observables en la ejecución de los estudiantes reflejen, con precisión, distinciones significativas en los constructos utilizados en la medición. Tales marcos requieren involucrar factores que comprometan las creencias sobre la propia competencia como estudiante (variables de autosistema), los de las habilidades cognitivas para aprender y los de autorregulación (para estar al tanto de la naturaleza de la tarea y para la administración de recursos necesarios y disponibles).

Ha sido práctica común que la medición de resultados de aprendizaje enfatice la función administrativa y descuide el control de calidad que la evaluación debe ejercer sobre ellos. Esto generó, por una parte, desconocimiento sobre qué es lo que aprenden los estudiantes en las escuelas, en particular, de los niveles reales en los que estrategias cognitivas y autorregulatorias, responsables de aprendizajes significativos y para toda la vida, son desarrollados en la práctica educativa y, por la otra, la imposibilidad de utilizar la evaluación como artefacto privilegiado para orientar la autorregulación del aprendiz en aspectos 
Motivación en línea y resultados del aprendizaje en estudiantes universitarios

significativos del aprendizaje (Castañeda, en prensa). En tanto la evaluación de resultados de aprendizaje es un componente central del proceso de aprender, su potencial para valorar y potenciar conocimientos y habilidades significativos, es indiscutible (Castañeda, en prensa).

Castañeda y Martínez (1999) conciben al desarrollo cognitivo como un factor importante de ser fomentado a partir de la instrucción. Según las autoras, construir esta nueva perspectiva requirió muchos cambios y ajustes entre la psicología del desarrollo y la psicología instruccional tradicional. Mencionan que los avances de la nueva psicología instruccional pueden establecerse, en términos generales, a partir de seis aspectos sustanciales de la teoría cognoscitiva contemporánea: 1) la noción constructivista del aprendizaje, 2) la noción del aprendizaje como cambio conceptual, 3) el conocimiento y el aprendizaje estratégico, 4) el conocimiento y las habilidades de autorregulación, 5) la cognición distribuida, y 6) la interfase afectivo-motivacional.

Considerando los seis aspectos anteriores, el área de desarrollo cognitivo (Castañeda \& López, 1994) enfatiza el trabajo del docente, el cual debe relacionarse estrechamente con el qué del currículo escolar (contenido) y con el cómo se enseña (mejores formas de adquirir información). En el ámbito de educación superior se discute la dificultad que tienen los alumnos para adquirir y aplicar los conocimientos que se les han impartido desde la educación básica hasta que egresan del nivel superior, así que su desempeño profesional no es tan eficiente como se requiere (Girardi, Arriola, Sagaón \& Montero-Kubli, 1999). Las mismas autoras (Girardi et al., 1999) mencionan que el estudio del comportamiento humano inteligente es un reto para los estudiosos de la educación. 
María de los Ángeles Maytorena N., Daniel González L. y Sandra Castañeda F.

\section{Evaluación del aprendizaje}

Se ha documentado que la enseñanza tradicional lleva a muchos profesores a evaluar principalmente el recuerdo de información (Martínez-Guerrero \& Sánchez-Sosa, 1993). Los estudiantes presentan exámenes memorísticos por lo que adoptan estilos superficiales de sobrevivencia escolar. Esto ocasiona que se presenten dificultades para aprender sus materias, entre otras razones porque carecen de estrategias eficaces de estudio que les ayuden a comprender las clases, las lecturas asignadas y les posibiliten un avance académico satisfactorio (Castañeda, Lugo, Pineda \& Romero, 1998).

La calificación otorgada en una evaluación forma parte de un proceso complejo en el que intervienen esquemas y mecanismos cognitivos, además de características personales.

Según Castañeda y López (1999), los cambios requeridos en la educación superior en México no serán suficientes con el simple reemplazo de métodos y procedimientos de enseñanza. Se requiere una nueva concepción del aprendizaje donde la comprensión, el pensamiento, la solución de problemas y el conocimiento reflexivo se conciban como interdependientes del contenido aprendido, de las habilidades intelectuales desarrolladas durante la formación y de los diversos contextos de aprendizaje. También se requiere de un proceso de evaluación entendido como "la aplicación sistemática de la metodología de la investigación de las ciencias sociales para estimar la conceptualización, diseño, implementación y utilidad de los programas sociales de intervención" (Rossi \& Freeman, 1993, p.5). La evaluación es un proceso sumamente importante en la adecuación y transformación que requiere la educación superior.

Un sistema de evaluación de los aprendizajes se concibe como un proceso cíclico que se desarrolla durante todo el proceso de enseñanza-aprendizaje por lo que para el proceso de evaluación deben 
Motivación en línea y resultados del aprendizaje en estudiantes universitarios

tenerse claros: 1) los objetivos (metas y propósitos) y estrategias metodológicas que están permitiendo el desarrollo de competencias, habilidades, destrezas, actitudes y valores; 2) las variables que intervienen en el proceso de aprendizaje y que facilitan y dificultan el logro de los objetivos planteados; 3) los tipos y formas de procedimientos más adecuados para realizar la evaluación de los aprendizajes propuestos; y 4) la necesidad de mantener un intercambio de experiencias mediante trabajos en equipo (Barrios, 2000, p. 289).

La educación superior incorpora los nuevos objetivos del aprendizaje que se derivan de investigaciones realizadas acerca del desarrollo de la pericia y, en esa medida, se requiere la generación de modelos y procedimientos de medición de resultados del aprendizaje que posibiliten guiar el diseño de exámenes que satisfagan lo que los objetivos demandan (Castañeda \& López, 1999).

Este enfoque también concibe la posibilidad de evaluar el aprendizaje y la modificación de actitudes considerando la necesidad de poseer instrumentos y técnicas poderosas para valorar con veracidad la forma en que éstas se expresan ante objetos, personas o situaciones. Existen dos aproximaciones para la evaluación de actitudes de los alumnos que realiza el profesor. En la autoevaluación, una de las técnicas utilizadas son los instrumentos de autoreporte como escalas y cuestionarios (Hernández, 1998).

La aproximación cognoscitiva a la evaluación del aprendizaje se basa en describir claramente el conocimiento semántico, procedimental y estratégico que se requiere para realizar una determinada actividad. De esta manera, la importancia de la evaluación con enfoque cognoscitivo se encuentra en la posibilidad de obtener niveles de análisis (Castañeda, 2000) y de detalle hasta ahora no disponibles, lo que permite definir con precisión aspectos incorrectos o incompletos del conocimiento del alumno, así como estrategias específicas que permitan intervenir al respecto. 
María de los Ángeles Maytorena N., Daniel González L. y Sandra Castañeda F.

Con relación a lo anterior, Herman (1998) menciona además que es necesario considerar el contenido y la naturaleza de la evaluación que puede contribuir a mejorar el aprendizaje de los estudiantes. La concepción que subyace a este enfoque es la suposición de que si la ejecución humana es entendida, podremos probarla y diagnosticarla, por lo que también permite encontrar la forma de elaborar estrategias para mejorarla. Sin embargo, no debe olvidarse que la ejecución de cualquier tarea no es función únicamente de habilidad intelectual; intervienen también otros factores. En los resultados de aprendizaje se involucran además de las cognitivas, otras variables que intervienen en el desempeño académico de los estudiantes. El aprendizaje y la enseñanza poseen también elementos afectivo-motivacionales y sociales. Esto hace más difícil el proceso de evaluación debido a que, en opinión de Glaser (1998), los sistemas de evaluación que se proponen dependen del conocimiento sobre cómo se aprenden ciertos conocimientos y habilidades.

Evaluar las autovaloraciones que los estudiantes realizan sobre su competencia subjetiva, sobre su estado emocional al iniciar o terminar una tarea, tanto como la utilidad percibida de la misma se fundamenta en el hecho de que es la percepción de los estudiantes sobre sí mismos, más que la percepción que otros tengan sobre ellos (profesores y terceros interesados), la que predice la motivación y el logro del estudiante (McCombs, 1998, 1999). Tratándose de actividades constructivas autorreguladas, es central entender las autovaloraciones que los aprendices generan en tanto éstas aclaran las creencias que tienen acerca de cómo se visualizan a sí mismos, de cómo visualizan a otros y de qué tanta libertad o control son percibidos en la interacción entre ellos y los otros.

\section{Motivación del aprendizaje}

Durante las últimas tres décadas (Good \& Brophy, 1995; López, Castañeda \& Orozco, 1989) se ha observado un progreso considerable 
en el estudio de los aspectos generales de la motivación del aprendizaje, cuya pertinencia estriba en la conceptualización del aprendizaje como un proceso activo que supone un esfuerzo propositivamente orientado a la obtención de resultados planeados (Gómez, Gúma, González \& Santiago, 1999), los que varían según la intensidad y modalidad que se adopte en determinada situación.

El estudio de la motivación, según Aguilar, Martínez, Valencia, Conroy y Girardo (1997), dejó de ser externo e interno para centrarse en procesos cognitivos y afectivos caracterizados por la identificación de patrones motivacionales formados por conjuntos organizados de metas, estrategias de afrontamiento, expectativas de éxito y grados de persistencia, que trascienden a distintas situaciones.

Los mismos autores opinan que el patrón motivacional típico interno de las metas de maestría se caracteriza por la concentración en la tarea y el aprendizaje, motivación intrínseca, atribuciones de esfuerzo, uso de estrategias efectivas de aprendizaje y de autorregulación. Mientras que el patrón motivacional asociado a las metas de evitación del trabajo es caracterizado por elementos negativos tales como distracción y falta de concentración en la tarea, atribuciones de capacidad, estrategias de aprendizaje ineficientes, baja persistencia y afecto negativo.

Por lo anterior, se considera que las atribuciones o causas percibidas por los resultados de la tarea influyen sobre las creencias y las conductas de logro (Schunk, 1990), las cuales se miden por las representaciones y percepciones que tienen los individuos de la tarea tales como capacidad, esfuerzo, dificultad de la tarea, suerte (Aguilar et al., 1997) y del contexto social en que se realiza dicha tarea. El factor motivacional medido en estudiantes universitarios se ha hecho a través de las creencias de control; además, el fracaso es atribuido a la falta de esfuerzo (Roces, Tourón \& González, 1995). Otros autores lo atribuyen también a la distracción (Schunk, 1990), a la falta de 
María de los Ángeles Maytorena N., Daniel González L. y Sandra Castañeda F.

concentración en la tarea (Aguilar et al., 1997), a las atribuciones de capacidad (Roces, Tourón \& González, 1995), a estrategias de aprendizaje ineficientes (Manassero \& Vázquez, 1995), a la baja persistencia (Good \& Brophy, 1995), y al afecto negativo. Mientras que el éxito lo atribuyen al entendimiento y comprensión de los contenidos (Roces, Tourón \& González, 1995), los estudiantes pueden conseguir lo que desean, siempre y cuando inviertan el esfuerzo necesario.

\section{Objetivos}

Considerando los planteamientos anteriormente expuestos, se investigó cómo influye el estado motivacional al realizar una tarea académica específica en estudiantes de licenciatura. Así, se plantearon los siguientes objetivos:

- Comparar cómo influye el grado de motivación que presentan los estudiantes antes y después de realizar una tarea de evaluación sobre conocimientos de Metodología de la Investigación.

- Comparar la ejecución de los estudiantes de cuatro licenciaturas universitarias en una tarea de evaluación correspondiente a la competencia metodológica durante el primer y tercer semestre de licenciatura.

\section{Metodología}

\section{Tipo de estudio}

Este es un estudio de varias cohortes, observacional y comparativo, debido a que la muestra quedó conformada por carreras universitarias de dos divisiones distintas. 
Motivación en línea y resultados del aprendizaje en estudiantes universitarios

\section{Participantes}

La muestra del estudio fue una muestra disponible, no aleatoria, conformada por 186 estudiantes de las licenciaturas en Psicología (53), Trabajo Social (45), Medicina (54) y Enfermería (34). De los estudiantes que conformaron la muestra, 108 se encontraban inscritos en primer semestre y el resto (78) en tercero. La edad promedio de los estudiantes entrevistados fue de 19.7 años $(D E=3.8)$ quienes reportaron un ingreso mensual familiar promedio de $\$ 6,488.72$ ( $D E=$ 5137.70). El 79\% de la muestra fueron mujeres; $94 \%$ de ellos eran solteros y $84 \%$ no trabajaban.

\section{Instrumentos}

1) La Prueba de Metodología General (Maytorena, 2003), que mide la competencia metodológica (definida como el dominio y la pericia que el alumno debe mostrar para comprender y aplicar los principios básicos de los métodos de investigación en general), construida a partir del Modelo Multidimensional de Evaluación propuesto por Castañeda (1998) y validada en un estudio anterior (Maytorena, González \& Castañeda, 2003). Contiene 27 reactivos de opción múltiple con cuatro opciones de respuesta, una respuesta correcta y tres respuestas incorrectas como distractores verosímiles.

2) El Instrumento de Motivación en Línea (Boekaerts, 1991), tanto en su porción anterior como posterior a la tarea, que fue adaptado en México por Castañeda (1996). El Cuestionario Anterior a la Tarea contiene 28 reactivos tipo Likert. Es un conjunto de respuestas que mide seis subescalas relacionadas con el estado afectivo-motivacional previo a la ejecución de una tarea académica específica y que van a influir, ya sea de forma positiva o negativa, en la manera en que se ejecuta la misma. Las escalas que conforman la porción de motivación a la tarea son: estado emocional, atractividad de la tarea, competencia subjetiva, utilidad percibida sobre la tarea, intención de aprendizaje y ansiedad de estado. 
El Cuestionario Posterior a la Tarea es un conjunto de 35 reactivos tipo Likert, que mide siete subescalas relacionadas con su estado afectivo-motivacional, luego de haber terminado la tarea que le fue asignada. Las escalas que lo componen son: estado emocional, atractividad de la tarea, competencia subjetiva, utilidad percibida sobre la tarea, esfuerzo reportado, evaluación del resultado y atribución después del éxito o del fracaso.

3) Una Ficha de Datos Sociodemográficos con el fin de identificar datos económicos, familiares y sociales de los alumnos de Psicología, Trabajo Social, Medicina y Enfermería que conformaron la muestra del estudio.

\section{Procedimiento}

Se solicitó permiso para acudir a los salones de clase correspondientes y realizar la aplicación de los cuestionarios y la prueba de ejecución académica. Al dar las instrucciones se aclaró que esta tarea no afectaría sus calificaciones. Primero se les proporcionó la Ficha de Datos Sociodemográficos y el Cuestionario Anterior a la Tarea enfatizando que, en este último, las preguntas se respondieran pensando en que estuvieran a punto de realizar una prueba de Metodología General. Cuando terminaron de responder dichos cuestionarios, se les proporcionó la prueba de ejecución con el dominio general de Metodología. Por último, respondieron el Cuestionario Posterior a la Tarea.

\section{Análisis y síntesis de datos}

Se realizaron análisis estadísticos descriptivos para las variables sociodemográficas y análisis multivariados para conocer el nivel de ejecución en las tareas de cada licenciatura que conformó la muestra de estudio y su relación con la motivación. Estos análisis se realizaron en el programa estadístico SAS. También se realizó un análisis 
factorial confirmatorio (AFC) con el fin de probar el modelo teórico propuesto.

Para el análisis de las relaciones entre variables (AFC) se utilizó un modelo causal de ecuaciones estructurales (Bentler, 1995). La prueba del modelo incluyó la medición de bondad de ajuste entre el modelo inclusivo y el modelo restringido (o modelo propuesto). El modelo inclusivo refiere una interrelación total de factores y variables observadas, y a pesar de que se acepte que este tipo de relaciones existe aunque sea en forma mínima en la realidad, en ciencia se buscan, sobre la base del principio de parsimonia, modelos simples que expliquen lo más posible.

Para contrastar ambos modelos se utiliza el estadígrafo de $X^{2}$, el cual compara el grado de diferencias entre dos modelos. Aquí una $X^{2}$ alta y significativa refiere que los dos modelos son diferentes, por lo cual debe buscarse una $X^{2}$ no significativa, es decir, que su probabilidad asociada sea mayor a 0.05 , de tal manera que nos muestre que el modelo restringido no es diferente del modelo inclusivo, en términos de poder explicativo.

Otros índices reutilizados son el Índice Bentler-Bonet de Ajuste Normado (IBBAN), el Índice Bentler-Bonet de Ajuste No Normado (IBBANN) y el Índice de Ajuste Comparativo (IAC), incluidos dentro del programa EQS (Bentler, 1995). Estos índices producen resultados que van de 0 a 1.0 y se acepta .90 como índice de ajuste adecuado (Corral, 1995; Corral, Frías \& González, 2001).

\section{Resultados}

Se preguntó a los estudiantes de la muestra cuántas y cuáles actividades realizan dentro de la universidad además de asistir a clases, con el propósito de formar un factor llamado actividades extras y tratar de relacionarlo con la puntuación obtenida en la prueba de metodología. Se encontró que el $53.8 \%$ de la muestra no realiza 
María de los Ángeles Maytorena N., Daniel González L. y Sandra Castañeda F.

ninguna actividad extraescolar. De los estudiantes que sí realizan alguna actividad, lo que hacen generalmente es asistir a la biblioteca o al centro de cómputo.

Sólo $24.2 \%$ de la muestra pertenece a algún grupo. De ese porcentaje el $45 \%$ pertenece a un grupo religioso, el $27.5 \%$ a uno deportivo, el $12.5 \%$ a uno comunitario y el resto pertenece a un grupo artístico o a uno académico ( $7.5 \%$ para cada uno).

Con el objetivo de crear un factor denominado distractores escolares se preguntó a los estudiantes acerca de los días que visitan a sus amigos y el $28 \%$ dijo que los visitan dos días. Ante la pregunta de cuántos días de la semana van a fiestas, $33 \%$ dijo ir un día a fiestas y $10.4 \%$ dijo asistir un día a bares. En promedio ven 1.83 horas de televisión $(D E=1.58)$ y entran a internet en promedio 1.18 horas al día $(D E=1.62)$.

En el Cuadro 1 se presentan las frecuencias y porcentajes de las respuestas correctas a la prueba de Metodología General. La frecuencia menor de aciertos fue de 12 en el reactivo que les solicitaba elaborar una ficha de paráfrasis, mientras que la mayor frecuencia de aciertos fue de 141 en el reactivo que les requería identificar las características de la investigación científica. En dicho cuadro se muestra también que los conocimientos mejor dominados por los estudiantes de la muestra son: las características de la investigación científica, las etapas de la investigación científica, la definición de método científico, la definición de hipótesis, la definición de experimentación y la característica de que la ciencia es verificable.

Por otro lado, los contenidos con menor dominio por los estudiantes de la muestra fueron: la identificación de una ficha de paráfrasis y de una ficha textual, a qué se refiere la verificación de variables en el conocimiento científico, la formulación de una hipótesis, la elaboración de una ficha de paráfrasis, la definición 
Motivación en línea y resultados del aprendizaje en estudiantes universitarios

de ley científica y de inducción, y la elaboración de una ficha de síntesis.

\section{Cuadro 1}

Frecuencias y porcentajes de respuestas correctas en la prueba de metodología general

\begin{tabular}{llcc}
\hline Reactivo & $f$ & $\%$ \\
\hline R1 & Elementos ficha & 97 & 52.2 \\
R2 & Teoría & 120 & 64.5 \\
R3 & Deducción & 92 & 49.5 \\
R4 & Método científico & 127 & 68.3 \\
R5 & Ciencia & 64 & 34.4 \\
R6 & Hipótesis & 116 & 62.4 \\
R7 & Características de la investigación & 141 & 75.8 \\
R8 & Investigación científica & 73 & 39.2 \\
R9 & Experimentación & 112 & 60.2 \\
R10 & Describe resultados & 64 & 34.4 \\
R11 & Controlar condiciones & 27 & 14.5 \\
R12 & Ficha de trabajo & 90 & 48.4 \\
R13 & Ley científica & 65 & 34.9 \\
R14 & Ejemplo de variable & 96 & 51.6 \\
R15 & Tres etapas de la investigación & 128 & 68.8 \\
R16 & Principales funciones de la ciencia & 74 & 39.8 \\
R17 & Verificable & 109 & 58.6 \\
R18 & Inducción & 46 & 24.7 \\
R19 & Formulación de un problema & 59 & 31.7 \\
R20 & Ley científica & 46 & 24.7 \\
R21 & Formulación de hipótesis & 28 & 15.1 \\
R22 & Ficha textual-sin comillas & 14 & 7.5 \\
R23 & Ficha paráfrasis-con comillas & 12 & 6.5 \\
R24A & Tres elementos-una idea entrecomillada & 46 & 24.7 \\
R24B & Tres elementos-paráfrasis de ideas principales & 41 & 22.0 \\
R24C Tres elementos-opinión de ideas principales & 47 & 25.3 \\
R24D & Tres elementos-juicio/crítica de ideas principales & 66 & 35.5 \\
\hline
\end{tabular}

Se realizó una comparación de medias (prueba $t$ ) del número de aciertos en la prueba de Metodología General (Cuadro 2) con el propósito de identificar cuáles de los semestres obtuvo una mayor pun- 
María de los Ángeles Maytorena N., Daniel González L. y Sandra Castañeda F.

tuación total en la prueba de metodología. Se encontró que no hay diferencias significativas $(p<.05)$ en la puntuación obtenida por ambos grupos $(F=1.33)$.

\section{Cuadro 2}

Comparación de la puntuación total en metodología por semestre

\begin{tabular}{lccccc}
\hline Semestre & $N$ & $M$ & $D E$ & $t$ & $p>$ \\
\hline Primero & 108 & 37.81 & 4.04 & 1.65 & .09 \\
Tercero & 78 & 36.87 & 3.49 & & \\
\hline
\end{tabular}

De igual manera se creó una variable de Motivación Total, tanto anterior como posterior a la tarea, con el propósito de comparar el grado de motivación con el que iniciaron la tarea de Metodología y el grado de motivación con el que concluyeron la tarea. La motivación posterior se comparó en dos partes, una para los que percibieron éxito y otra para los que percibieron fracaso. Esta comparación de medias (prueba $t$ ) se realizó dividiendo al total de la muestra por sus resultados en la prueba de metodología. Los estudiantes que obtuvieron 38 puntos (mediana) o más fueron clasificados en grupo alto y los estudiantes que obtuvieron de 2 a 37 puntos, se ubicaron en el grupo bajo.

No se encontraron diferencias significativas en la motivación anterior. El Cuadro 3 muestra esos resultados; ambos grupos iniciaron la tarea con el mismo grado de motivación $(F=1.04)$.

\section{Cuadro 3}

Comparación de grupos alto/bajo en la motivación anterior a la tarea

\begin{tabular}{lccccc}
\hline Grupo & $N$ & $M$ & $D E$ & $t$ & $p$ \\
\hline Bajo & 92 & 4.35 & .72 & .06 & .9 \\
Alto & 94 & 4.35 & .71 & & \\
\hline
\end{tabular}

El Cuadro 4 muestra la comparación entre grupo alto/bajo en la motivación posterior a la tarea considerando a los estudiantes de la 
muestra que percibieron tener éxito en la tarea de metodología; la motivación de ambos grupos es significativamente $(p>.05)$ diferente $(F=1.18)$. Se muestra también la comparación por grupos de la motivación posterior de los estudiantes que percibieron fracaso en los resultados de la tarea; la diferencia $(F=1.07)$ entre ambos grupos es significativa $(p<.05)$.

\section{Cuadro 4}

Comparación de la motivación posterior por grupos alto/bajo en la situación de éxito y fracaso percibido

\begin{tabular}{|c|c|c|c|c|c|c|c|c|c|c|}
\hline \multirow[b]{2}{*}{ Grupo } & \multicolumn{5}{|c|}{ Éxito } & \multicolumn{5}{|c|}{ Fracaso } \\
\hline & $N$ & $M$ & $D E$ & $t$ & $p>$ & $N$ & $a$ & $D E$ & $t$ & $p$ \\
\hline Bajo & 92 & 3.47 & .59 & 2.38 & .01 & 92 & 3.51 & .61 & 2.60 & .00 \\
\hline Alto & 94 & 3.68 & .64 & & & 94 & 3.74 & .59 & & \\
\hline
\end{tabular}

Los estudiantes de Medicina (24.1\%) y Psicología (17.7\%) son los que se ubicaron en un mayor porcentaje en el grupo alto de la prueba de Metodología y los estudiantes de Enfermería (13.9\%) y Trabajo social (19.8\%) se ubicaron mayormente en el grupo bajo en la misma prueba. El valor de chi cuadrada en estos datos es 55.3 (3gl) y con una probabilidad asociada de $p<.001$.

El Cuadro 5 presenta el índice de consistencia interna (alfa de Cronbach) de las escalas que conforman el Cuestionario de Motivación Anterior. La escala Estado Emocional Anterior quedó conformada por ocho reactivos con una media de 4.32 y un índice de consistencia interna de .80 , medido en una escala diferencial semántico que va de 1 (Bajo Estado Emocional Anterior) a 6 (Alto Estado Emocional Anterior). La escala Atractividad de la Tarea quedó integrada por cuatro reactivos con una media de 3.76 y un alfa de Cronbach de .84 , medido en una escala diferencial semántico de 1 (Atractividad Baja) 
María de los Ángeles Maytorena N., Daniel González L. y Sandra Castañeda F.

\section{Cuadro 5}

Índice de consistencia interna (alfa de Cronbach) de las escalas que conforman el Cuestionario de Motivación Anterior

\begin{tabular}{|c|c|c|c|c|}
\hline Variable & $N$ & $M$ & $D E$ & Alfa de Cronbach \\
\hline Estado emocional anterior & 179 & 4.32 & & .80 \\
\hline Infeliz & & 4.41 & 1.28 & \\
\hline No aburrido & & 3.81 & 1.63 & \\
\hline No Satisfecho & & 4.06 & 1.43 & \\
\hline No triste & & 4.72 & 1.52 & \\
\hline No cansado & & 3.68 & 1.72 & \\
\hline No estoy harto & & 4.35 & 1.58 & \\
\hline No estoy de buen humor & & 4.43 & 1.67 & \\
\hline No estoy enojado & & 5.07 & 1.29 & \\
\hline Atractividad de la tarea & 183 & 3.76 & & .84 \\
\hline Nada de ganas & & 3.54 & 1.47 & \\
\hline Nada entusiasta & & 4.09 & 1.35 & \\
\hline Nada divertidas & & 3.28 & 1.45 & \\
\hline Creo que no lo disfrutaré & & 4.12 & 1.43 & \\
\hline Autoconfianza & 183 & 4.47 & & .77 \\
\hline Nada hábil & & 3.98 & 1.10 & \\
\hline Nada fácil & & 4.23 & 1.15 & \\
\hline Nada bien & & 4.92 & 0.95 & \\
\hline Casi nunca & & 4.60 & 1.27 & \\
\hline Nada difícil & & 4.18 & 1.35 & \\
\hline No necesito esforzarme & & 4.51 & 1.35 & \\
\hline Calificación insuficiente & & 5.18 & 0.95 & \\
\hline Estoy por debajo de mis & & & & \\
\hline compañeros & & 4.15 & 0.95 & \\
\hline Intención del aprendizaje & 184 & 4.71 & & .78 \\
\hline Sin atención & & 4.74 & 1.10 & \\
\hline Espero sólo pasar & & 5.02 & 1.16 & \\
\hline Me esforzaré un poco & & 4.75 & 1.33 & \\
\hline Empiezo y luego veo & & 4.35 & 1.39 & \\
\hline Utilidad percibida & 184 & 4.44 & & .71 \\
\hline Nada útil & & 4.17 & 1.36 & \\
\hline Nada importante & & 4.71 & 1.26 & \\
\hline
\end{tabular}


a 6 (Atractividad Alta). La escala de Autoconfianza se integró por ocho reactivos con una media de 4.47 y su índice de confiabilidad fue de .77 , medida en una escala diferencial semántico de 1 (Autoconfianza Baja) a 6 (Autoconfianza Alta). La escala Intención del Aprendizaje tuvo un alfa de .78 y una media de 4.71 , quedó integrada por cuatro reactivos medidos en una escala diferencial semántico que va de 1 (Baja Intención) a 6 (Alta Intención). Por último, la escala Utilidad Percibida se conformó por dos reactivos y obtuvo una media de 4.44 y un índice de consistencia interna de .71, medida en una escala de 1 (Utilidad Baja) a 6 (Utilidad Alta). La consistencia interna (alfa de Cronbach) del total de reactivos fue de .90 para el Cuestionario de Motivación Anterior.

En el Cuadro 6 se presentan los índices de consistencia interna (alfa de Cronbach) de los dos tipos de conocimiento que conformaron la prueba de Metodología General, así como las medias y desviaciones estándar de los reactivos.

La escala de Comprensión quedó conformada por 11 reactivos con una media de 1.51 y un índice de consistencia interna de .54 medida en una escala de 1 (Respuesta Incorrecta) a 2 (Respuesta Correcta); mientras que la escala de Solución de Problemas se integró por seis reactivos con una media de 1.20 y un alfa igual a .73, medida en una escala de 1 (Respuesta Incorrecta) a 2 (Respuesta Correcta). El cuestionario total alcanzó un índice de consistencia interna (alfa de Cronbach) de .71. 
María de los Ángeles Maytorena N., Daniel González L. y Sandra Castañeda F.

\section{Cuadro 6}

Índice de consistencia interna (alfa de Cronbach) de los niveles de dificultad que conforman el Cuestionario de Metodología General

\begin{tabular}{lccc}
\hline Variable & $M$ & $D E$ & Alfa de Cronbach \\
\hline Comprensión de lo aprendido & $I .51$ & & .54 \\
Elementos ficha & 1.47 & 0.50 & \\
Teoría & & 1.35 & 0.47 \\
Método científico & 1.68 & 0.46 & \\
Ciencia & 1.34 & 0.47 & \\
Características de la investigación & 1.75 & 0.42 & \\
Investigación científica & 1.39 & 0.48 & \\
Experimentación & 1.60 & 0.49 & \\
Ficha de trabajo & 1.48 & 0.50 & \\
Ley científica & 1.34 & 0.47 & \\
Tres etapas de la investigación & 1.68 & 0.46 & \\
Verificable & 1.58 & 0.49 & \\
Solución de problemas & 1.20 & & \\
Identificar ficha textual & 1.07 & 0.26 & \\
Identificar ficha paráfrasis & 1.06 & 0.24 & \\
Ficha textual & 1.24 & 0.43 & \\
Ficha paráfrasis & 1.22 & 0.41 & \\
Ficha síntesis & 1.25 & 0.43 & \\
Ficha comentario & 1.35 & 0.47 & \\
\hline
\end{tabular}

En el Cuadro 7 se presentan los reactivos que conforman las escalas del Cuestionario de Motivación Posterior con sus medias e índices de consistencia interna; dichos índices fueron superiores a .80. La escala Estado Emocional Posterior quedó integrada por 10 reactivos con una media de 4.07 y un índice de consistencia interna de .81, medida en una escala de 1 (Bajo Estado Emocional Posterior) a 6 (Alto Estado Emocional Posterior). La escala Esfuerzo Reportado se conformó por cuatro reactivos con una media de 3.80 y un alfa de .81 medidos en una escala de 1 (Bajo Esfuerzo) a 6 (Alto Esfuerzo). La escala Evaluación de la Tarea quedó integrada por dos reactivos medidos en una escala de 1 (Bajo Resultado) a 6 (Alto Resultado) con una media de 3.71 y un alfa de .84. Por otro lado, los reactivos 
Motivación en línea y resultados del aprendizaje en estudiantes universitarios

que miden Atractividad de la Tarea, Autoconfianza y Utilidad Percibida obtuvieron medias de $2.81,3.30$ y 4.41 , respectivamente.

\section{Cuadro 7}

Indice de consistencia interna (alfa de Cronbach) de las escalas del Cuestionario de Motivación Posterior

\begin{tabular}{lccc}
\hline Variable & Media & DE & Alfa de Cronbach \\
\hline Estado emocional posterior & 4.07 & & .81 \\
No aliviado & 4.43 & 1.45 & \\
Incómodo & 4.33 & 1.56 & \\
Sin nervios & 5.02 & 1.51 & \\
Insatisfecho & 4.31 & 1.56 & \\
No estoy harto & 4.11 & 1.66 & \\
Intranquilo & 4.37 & 1.73 & \\
Despreocupado & 4.56 & 1.66 & \\
Inseguro & 4.68 & 1.41 & \\
No molesto & 3.75 & 1.88 & \\
No angustiado & 4.88 & 1.45 & \\
Atractividad de la tarea & 2.81 & 1.42 & \\
Esfuerzo reportado & 3.80 & & .81 \\
Ninguna atención & 4.56 & 1.21 & \\
Empezaba pero no continuaba & 4.55 & 1.19 & \\
Me esforcé un poco & 4.51 & 1.31 & \\
Nada distraído & 3.93 & 1.65 & \\
Autoconfianza & 3.30 & 1.45 & \\
Evaluación de la tarea & 3.71 & & .84 \\
Insuficiente & 4.52 & 0.91 & \\
Muy mal & 4.54 & 1.04 & \\
Utilidad percibida & 4.41 & 1.43 & \\
\hline
\end{tabular}

Análisis factorial confirmatorio

La Figura 1 representa el modelo estructural de motivación en línea y los resultados en la tarea de metodología.

Se observa que la Motivación Anterior a la Tarea, variable latente conformada por los indicadores Estado Emocional Anterior, 
María de los Ángeles Maytorena N., Daniel González L. y Sandra Castañeda F.

con un peso factorial de .48; Competencia Subjetiva, con un peso factorial de .74 y Utilidad Percibida, con un peso factorial de .62, afecta de forma directa y significativa (peso factorial .33) a la variable puntuación total en la prueba de metodología (índice conformado por los reactivos de comprensión de lo aprendido y reactivos de solución de problemas). La $R^{2}$ de la variable puntuación total en la prueba de metodología es igual a .12, lo cual significa que su variabilidad es definida en un $12 \%$ por la motivación anterior.

A su vez, la puntuación total en la prueba de metodología afecta de manera directa y significativa $(p<.05)$ al constructo Motivación Posterior a la Tarea, variable latente construida por los indicadores Estado Emocional Posterior (peso factorial .58), Autoconfianza, con un peso factorial de .44 y Evaluación de la Tarea, cuyo peso factorial fue de .76. La $R^{2}$ de la Motivación Posterior es igual a .17, lo que significa que la puntuación en la prueba de metodología explica el $17 \%$ de variabilidad de la motivación posterior.

En el mismo modelo la variable latente distractores escolares, construida por las variables observadas días de la semana que visitan a los amigos (con un peso factorial de .62), horas diarias que ven televisión (con un peso factorial de .42), horas diarias que entran a internet (con un peso factorial de .80) y la variable días de la semana que van a bares (con un peso factorial de .58) no afectó significativamente $(p>.05)$ a la Puntuación Total en la prueba de metodología. El mismo efecto se observó para la variable Actividades Extracurriculares, la cual se construyó por la variable número de actividades que realiza en la universidad además de asistir a clases (con un peso factorial de 1.0) y las horas a la semana que dedica a cada una de esas actividades (con pesos factoriales de $.38, .50$ y .50 , respectivamente). Ninguna de las correlaciones entre las variables latentes que funcionan como variables independientes fue significativa. 


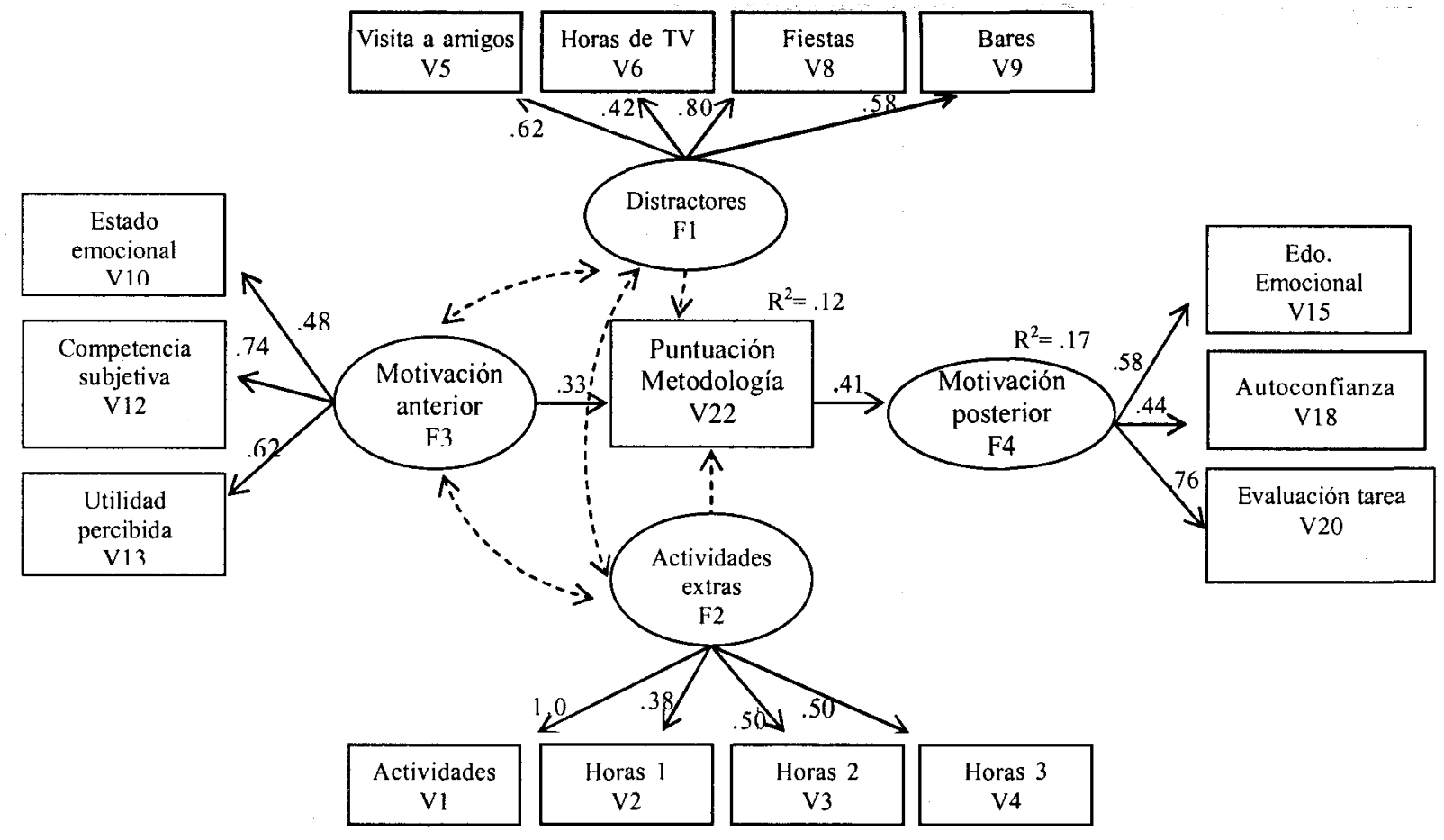

Figura 1. Modelo estructural de motivación en línea y resultados en Metodología General. $X^{2}=90(83 \mathrm{gl}) \mathrm{p}=.27 ;$ IBAN $=.67$ IBANN $=.95$ IAC $=.96$. 
María de los Ángeles Maytorena N., Daniel González L. y Sandra Castañeda F.

La $X^{2}$ del modelo fue igual a 90 con 83 grados de libertad y una probabilidad asociada igual a .27. El Indice Bentler-Bonet de Ajuste Normado fue igual a .67, el Indice Bentler-Bonet de Ajuste No Normado fue de .95 y el Indice de Ajuste Comparativo fue igual a .96. Estos datos muestran que el modelo tiene poder de explicación, es decir, que el modelo está respaldado por los datos (Bentler, 1995; Corral, 1995).

\section{Discusión}

Se elaboró y probó una prueba sobre contenidos de Metodología General que mide niveles de conocimiento (comprensión de lo aprendido y solución de problemas) en dos contextos de evaluación (el de reconocimiento y el de recuerdo).

Se comparó la ejecución de los estudiantes de las cuatro licenciaturas universitarias que conformaron la muestra en la tarea de evaluación correspondiente a su competencia metodológica durante los primeros semestres de licenciatura. Los resultados encontrados destacan que los estudiantes de las licenciaturas en Medicina y Psicología son los que más conocimientos básicos de metodología poseen, medidos con la prueba elaborada. No ocurre así con los estudiantes de las licenciaturas en Enfermería y Trabajo Social.

Al considerar sólo a los estudiantes que evaluaron su ejecución en la prueba de Metodología General como exitosa, se encontró que los estudiantes que obtuvieron una mayor puntuación en la prueba presentaron una motivación inicial significativamente mayor que los estudiantes que obtuvieron una puntuación baja en dicha prueba. En cuanto a los estudiantes que perciben su ejecución en la prueba de Metodología General como un fracaso, la diferencia en el grado de motivación también fue significativa. 
Al contrastar a los exitosos y a los fracasados en cuanto a la motivación posterior a la ejecución se observa que el grado de motivación con el que finalizaron la tarea resultó mayor que el grado de motivación con el que finalizaron la tarea los estudiantes que percibieron tener éxito. Estos resultados pueden deberse al hecho de que la motivación intrínseca puede incrementarse mediante el automonitoreo y se relaciona de forma directa con la autoeficacia (Aguilar, Valencia \& Martínez, 2002). Los resultados anteriores apoyan parcialmente lo dicho por Cazares (2003) acerca de que las variables motivacionales no predicen en forma directa la ejecución en pruebas de rendimiento en formato de opción múltiple como la utilizada en este trabajo.

Se logró modelar una estructura de motivación en línea y resultados en Metodología General. Los datos revelan que el estado afectivo-motivacional con el que se inicia una tarea predice de forma directa y positiva la ejecución en una tarea académica (explica el $12 \%$ de su varianza) y ésta, a su vez, influye en el estado afectivomotivacional posterior (explicando el $17 \%$ de su varianza). Estos resultados indican que es importante considerar los factores afectivomotivacionales en el contexto de los resultados del aprendizaje como lo afirman Castañeda, Lugo, Pineda y Romero (1998), Cazares (2003), Pintrich (1998) y Reinosa (1998), corroborando que además de estudiar el aspecto cognitivo, es necesario estudiar también el factor motivacional con el fin de determinar el papel que dicho factor tiene en la investigación educativa y estar en posibilidad de superar las criticas hechas a los estudios desde la perspectiva de la cognición dura, que sólo contempla dimensiones cognoscitivas del aprendizaje y deja fuera el estudio de los efectos de la dimensión afectivomotivacional, con las implicaciones inherentes.

El modelo estructural resultante muestra que las variables que mejor predijeron los resultados en la prueba de Metodología General desarrollada fueron el estado emocional anterior, la competencia 
María de los Ángeles Maytorena N., Daniel González L. y Sandra Castañeda F.

subjetiva y la utilidad percibida, mientras que las mejores variables posteriores fueron el estado emocional posterior, la autoconfianza y la evaluación de la tarea. Tales resultados representan una contribución a las investigaciones sobre motivación y apoyan la importancia del estudio de la motivación en la ejecución de una tarea académica, debido a que ésta se define como el interés y el disfrute en una actividad/tarea por sí misma (Aguilar, Valencia, Martínez \& Vallejo, 2002).

Contrastando los resultados obtenidos en este trabajo con la definición antes expuesta, se corrobora la importancia del estado emocional, tanto anterior como posterior a la tarea, variables que aportaron un peso factorial importante en este modelo (.48 y .58 respectivamente). Las variables competencia subjetiva, autoconfianza y evaluación de la tarea corresponden a la percepción de la persona de sí misma. Estas variables proporcionan información acerca del estudio de la competencia percibida, la cual ha sido evaluada por muy pocos estudios (Aguilar et al., 2002), y representa el grado en que una persona cree que puede realizar bien una tarea, por lo que aumentando la autoeficacia a través de la solución de problemas en complejidad creciente (como el ejemplo de la prueba de Metodología utilizada en esta investigación), puede incrementar el interés en una materia o un trabajo académico. Tales variables corresponden también con el grado en que la persona se esmera por realizar bien una actividad, lo cual ha sido considerado un importante mediador en la motivación intrínseca (Aguilar et al., 2002).

Otra variable que resultó con importante poder de predicción (peso factorial de .62) en esta investigación fue la utilidad percibida; que corresponde teóricamente con el valor de la tarea la cual también se relaciona con el interés intrínseco (Aguilar et al., 2002).

La información antes presentada acerca de las variables que mejor predicción tienen hacia los resultados del aprendizaje corresponde a 
la motivación intrínseca, la cual se ha relacionado con el esfuerzo y la dedicación en una actividad académica. La motivación hacia un actividad no sólo se define por la preferencia que se tenga hacia ella, sino también por el tiempo que se invierte en su ejecución y los conocimientos que se acumulan sobre tal actividad, para que así demuestre confianza en hacer las cosas, motivación para aprender, esfuerzo en la realización de su trabajo, responsabilidad en el cumplimiento de sus actividades y capacidad para resolver problemas (Serna \& Canales, 1996). Los resultados encontrados a partir de la aplicación de la prueba de Metodología General no reflejaron diferencias entre los estudiantes de primer semestre y los estudiantes de tercer semestre que conformaron la muestra en cuanto a conocimientos de Metodología General se refiere (medidos por la prueba utilizada en esta investigación). Tal resultado refleja que los alumnos de la muestra no amplían los conocimientos de metodología con los que ingresan a la universidad en los semestres subsecuentes. Tal suposición nos ubica en la interrogante sobre el trabajo de los docentes de metodología y sobre el proceso de selección de los alumnos que se está llevando a cabo, debido a que las licenciaturas seleccionadas para participar en el estudio mencionan, dentro de su perfil de ingreso, que los aspirantes a dichas áreas deberán contar con conocimientos básicos de metodología y los resultados encontrados no reflejan el requerimiento establecido en el perfil de ingreso en lo referente al conocimiento sobre Metodología General.

\section{Recomendaciones}

- Se requiere de mayor investigación educativa sobre las variables consideradas distractores académicos debido a que su relación con la puntuación total en la prueba de metodología no fue significativa. Sin embargo, la exposición excesiva a la televisión tiene consecuencias negativas en el rendimiento académico (Meyer, 1987, como se cita en González, Corral, Miranda \& Frías, 1998). 
María de los Ángeles Maytorena N., Daniel González L. y Sandra Castañeda F.

- Lo mismo se requiere para las variables relacionadas con las actividades extraescolares, aunque el hecho de que esta relación no sea significativa puede deberse quizás a que son muy pocos los estudiantes de la muestra que reportaron realizar actividades extracurriculares dentro de la universidad, además de asistir a clases.

- Los datos de este trabajo reflejan la influencia de la motivación en tareas generales como lo es Metodología; sin embargo, es necesario seguir investigando las variables afectivo-motivacionales asociadas a variables cognitivas para conocer la influencia que la motivación puede tener en otro tipo de tareas más específicas.

- El diseño de estrategias instruccionales que fomenten la motivación de los estudiantes hacia las actividades académicas se torna importante y necesario a partir de los resultados encontrados, debido a que, como los resultados del presente trabajo lo reflejan, los niveles altos de motivación están relacionados con resultados de aprendizaje de calidad.

- Una forma de fomentar o incrementar los niveles de motivación en el salón de clase puede ser a través de que el maestro relacione los contenidos del curso con actividades reales que el estudiante deberá desarrollar, es decir, hacer significativo el aprendizaje; o bien, que los estudiantes "aprendan haciendo", para que así desarrollen las habilidades y el interés por la tarea o contenido que se le imparta.

\section{Referencias}

Aguilar, J., Martínez, M., Valencia, A., Conroy, C. \& Girardo, A. (1997). Metas de logro, competitividad y perfiles motivacionales entre estudiantes universitarios. Revista Latina de Pensamiento y Lenguaje, 5, 25-35.

Aguilar, J., Valencia, A. \& Martínez, M. (2001-2002). Un modelo de las interrelaciones entre disposiciones motivacionales generales y específicas. Revista Latina de Pensamiento y Lenguaje, 10,119-127. 
Motivación en línea y resultados del aprendizaje en estudiantes universitarios

Aguilar, J., Valencia, A., Martínez, M. \& Vallejo, A. (2002). Un modelo estructural de la motivación intrínseca en estudiantes universitarios. En A. Bazán \& A. Arce (Eds.), Estrategias de Evaluación y Medición del Comportamiento (pp. 165-185). Obregón, Sonora: ITSON-UADY.

Barrios, O. R. (2000). Sistema de evaluación de los aprendizajes. En S. De la Torre \& O. Barrios, Estrategias didácticas innovadoras (pp. 289-293). México: Octaedro.

Bentler, P. (1995). EQS. Structural equations program manual. Los Angeles: BMDM Statistical Software INC.

Boekaerts, M., Pintrich, P. \& Zeidner, M. (2000). Handbook of selfregulation. San Diego: Academic Press.

Castañeda, S. (1993). Procesos cognitivos y educación médica. México: UNAM.

Castañeda, S. (1996). Interfase afectivo-motivacional en la comprensión de textos: estudio transcultural México-Holanda. Revista Latina de Pensamiento y Lenguaje, 4, 169-185.

Castañeda, S. (1998). Evaluación de resultados de aprendizaje en escenarios educativos. Revista Sonorense de Psicología, 12, 57- 67.

Castañeda, S. (2000). Análisis cognitivo de tareas. Laboratorio de Desarrollo e Innovación Tecnológica Posgrado, Universidad Nacional Autónoma de México, México.

Castañeda, S. (2002). A cognitive model for learning outcomes assessment. International Journal of Continuing Engineering Education and Life-long Learning, 12(1-4), 106.

Castañeda, S. (en prensa). Evaluación y fomento de la actividad constructiva y autorregulatoria, en actividades educativas ordinarias. En V. Alcaraz (Ed.), La investigación del comportamiento en México.

Castañeda, S. \& López, M. (1994). La nueva concepción de la inteligencia y su impacto en el desarrollo intelectual de los universitarios. México: Mecanograma.

Castañeda, S. \& López, M. (1999). Elaboración de un instrumento para la medición de conocimientos y habilidades en estudiantes 
María de los Ángeles Maytorena N., Daniel González L. y Sandra Castañeda F.

de psicología. Revista Intercontinental de Psicología y Educación, 1, 9-15.

Castañeda, S., Lugo, E., Pineda, L. \& Romero, N. (1998). Estado del arte de la evaluación y el fomento del desarrollo intelectual en la enseñanza de ciencias, artes y técnicas. En S. Castañeda (Ed.), Evaluación y fomento del desarrollo intelectual en la enseñanza de ciencias, artes y técnicas: perspectiva internacional en el umbral del siglo XXI (pp. 17-158). México: Porrúa-UNAMCONACYT.

Castañeda, S. \& Martínez, R. (1999). Enseñanza y aprendizaje estratégicos. Modelo integral de evaluación e instrucción. Revista Latina de Pensamiento y Lenguaje, 4, 251-278.

Cazares, A. (2003). Modelamiento de variables cognitivas, afectivomotivacionales y contextuales asociadas al aprendizaje académico. En D. González (Ed.), Modelamiento estructural en las ciencias sociales (Cap. 4). Hermosillo, Sonora: Unison.

Corral, V. (1995). Modelos de variables latentes para la investigación conductual. Acta Comportamentalia, 3, 171-190.

Corral, V., Frías, M. \& González, D. (2001). Análisis cuantitativo de variables latentes. Hermosillo, Sonora: Unison.

De Corte, E. (1995). Fostering cognitive development. A perspective from research on mathematics learning and instruction. Educational Psychologist, 30, 37-46.

Girardi, C., Arriola, M., Sagaón, A. \& Montero-Kubli, D. (1999).

Perfil cognoscitivo y de personalidad de estudiantes de licenciatura de la Universidad Intercontinental. Revista Intercontinental de Psicología y Educación, 1, 62-79.

Glaser, R. (1998). Pericia y evaluación. En M. Wittrock \& E. Baker (Eds.), Test y cognición: investigación cognitiva y mejora de las pruebas psicológicas (pp. 35-51). Barcelona: Paidós Ibérica.

Glaser, R. \& Baxter, G. P. (2002). Cognition and construct validity: Evidence for the nature of cognitive performance in assessment situations. En H. I. Braun, D. N. Jackson \& D. E. Wiley (Eds.), The role of constructs in psychological and educational measurement (pp. 179-227). Nueva Jersey: LEA. 
Gómez, F., Gúma, E., González, A. \& Santiago, J. (1999). Influencia de la motivación extrínseca sobre la ejecución de una tarea de memoria de trabajo en adultos jóvenes. Revista Latina de Pensamiento y Lenguaje, 7, 157-174.

González, L. D. (2001). Un modelo estructural de desempeño escolar en estudiantes de licenciatura. Tesis doctoral no publicada, Universidad Nacional Autónoma de México, México.

González, L. D., Corral, V., Miranda, J. \& Frías, M. (1998). Relaciones entre variables de apoyo familiar, esfuerzo académico y rendimiento escolar en estudiantes de secundaria: un modelo estructural. Enseñanza e Investigación en Psicología, 3, 163-183.

Good, T. \& Brophy, J. (1995). Psicología educativa contemporánea. México: McGraw Hill.

Herman, J. L. (1998). Investigación en cognición y aprendizaje: implicaciones para la práctica en la aplicación de pruebas de rendimiento. En M. Wittrock \& E. Baker (Eds.), Test y cognición: investigación cognitiva y mejora de las pruebas psicológicas (pp. 203-216). Barcelona: Paidós Ibérica.

Hernández, R. G. (1998). Paradigmas en psicología de la educación. México: Paidós.

López, M., Castañeda, S., Gómez, T. \& Orozco, C. (1989). Evaluación metacurricular en una población de estudiantes de educación superior: aspectos motivacionales. En Antología: la psicología cognoscitiva del aprendizaje (pp. 147-156). México: UNAM. Manassero, M. A. \& Vázquez, A. (1995). Dimensionalidad de las causas percibidas en situaciones de éxito y fracaso escolar. $R e$ vista de Psicología Social, 10, 235-255.

Martínez-Guerrero, J. \& Sánchez-Sosa, J. J. (1993). Estrategias de aprendizaje: análisis predictivo de hábitos de estudio en el desempeño académico de alumnos de bachillerato. Revista Mexicana de Psicología, 10, 63-73.

Maytorena, M. (2003). Evaluación en línea de resultados del aprendizaje de metodología en estudiantes de licenciatura. Tesis de maestría no publicada, Universidad de Sonora, México. 
María de los Ángeles Maytorena N., Daniel González L. y Sandra Castañeda F.

Maytorena, M., González, L. D. \& Castañeda, S. (2003). Diseño y validación de una prueba de metodología de la investigación para ingresantes universitarios. Investigaciones Educativas en Sonora, 5, 137-150.

McCombs, B. L. (1998). Integrating metacognition, affect and motivation in improving teacher education. En B. L. McCombs \& N. Lambert (Eds.), Issues in school reform: Psychological perspectives on learner-centered schools (pp. 379-408). Washington, DC: APA Books.

McCombs, B. L. (1999). What role does perceptual psychology play in educational reform today? En H. J. Freiberg (Ed.), Perceiving, behaving, becoming: Lessons learned (pp. 148-157). Alexandria, VA: Association for Supervision and Curriculum Development.

Pintrich, P. (1998). El papel de la motivación en el aprendizaje académico autorregulado. En S. Castañeda (Ed.), Evaluación y fomento del desarrollo intelectual en la enseñanza de las ciencias, artes y técnicas: perspectiva internacional en el umbral del siglo XXI (pp. 229-262). México: Porrúa-UNAM.

Reinosa, G. L. (1998). Identificación de predictores cognitivos y afectivo-motivacionales en la ejecución de tareas académicas, en estudiantes de nuevo ingreso a la Universidad Tecnologica de Nezahualcoyotl. Tesis de maestría no publicada, Universidad Nacional Autónoma de México, México.

Roces, C., Tourón, J. \& González, M. C. (1995). Motivación, estrategias de aprendizaje y rendimiento de los alumnos universitarios. Bordón, 47, 107-120.

Rossi, P. H. \& Freeman, H. E. (1993). Evaluation: A systematic approach. Newbury Park, CA: SAGE Publications.

Schunk, D. (1990). Formulación de metas y autoeficacia durante el aprendizaje autorregulado. Educational Psychologist, 25, 71-86.

Serna, M. L. \& Canales, A. (1996). Desafíos actuales y prospectivas del desarrollo de la educación. Revista de Psicología y Educación, 3, 30-33. 